\title{
Pulmonary Embolism as a Cause of Death in Psychiatric Inpatients: a Case Series
}

\author{
CC Lee, R Fung, SW Pang, TL Lo
}

\begin{abstract}
We report four cases of fatal pulmonary embolism confirmed by autopsy among inpatients in a Hong Kong psychiatric hospital from 2010 to 2014. None of the four patients had a medical or premorbid condition associated with vascular thromboembolism or causing prolonged immobilisation. Only two patients were taking long-term antipsychotic medication, but all were physically restrained shortly before the event.
\end{abstract}

CC Lee, MRCP, FRCPsych, Department of Psychiatry, Kwai Chung Hospital, Hong Kong

Regina Fung, BSc, MBA(HCM), Department of Psychiatry, Kwai Chung Hospital, Hong Kong

SW Pang, BSc (Hons), MSc (HCM), Department of Psychiatry, Kwai Chung Hospital, Hong Kong

TL Lo, FRCPsych, FHKAM(Psych), Department of Psychiatry, Kwai Chung Hospital, Hong Kong

Address for correspondence: Chi Chiu Lee, Department of Psychiatry, Kwai Chung Hospital, Hong Kong

Email:lccz04@ha.org.hk

Submitted: 9 April 2018; Accepted: 16 October 2018

\section{Introduction}

Vascular thromboembolism (VT) and pulmonary embolism (PE) are uncommon in Chinese people. In Hong Kong, the annual incidence of PE is 3.9 per 100000 population, and that of deep vein thrombosis (DVT) is 17.1 per 100000 population. ${ }^{1}$ However, the incidence of VT has been increasing since 1980s. A review of autopsies found that the incidence of PE in Hong Kong increased from $0.58 \%$ to $2.08 \%$ from 1979 to 1989 , and to $4.7 \%$ in 1994.2

Psychiatric patients are at risk of VT because of the features of catatonia, stupor, and immobilisation. The use of physical restraint and antipsychotics as well as metabolic complications such as diabetes mellitus and obesity also increase the risk. In a review of 14000 autopsy records from 1970 to 1994 in the Netherlands, 10 of 27 cases of PE involved psychiatric patients. ${ }^{3}$ In a retrospective analysis of $>28$ million adult patients in a US hospital, antipsychotic users had higher risk of PE. ${ }^{4}$

Physical restraint is a controversial practice in psychiatric care for preventing injury and reducing patient agitation. Physically restrained patients are prone to VT, and two such cases were reported in Norway. ${ }^{5}$ In Hong Kong, at least $39.7 \%$ of patients admitted to an acute psychiatric ward have been restrained. ${ }^{6}$ We reviewed all cases of inpatient mortality from 2010 to 2014 in a Hong Kong psychiatric hospital through the Advance Incident Reporting System.
Among 19572 admissions (9877 were men), about 8000 episodes per year involved physical restraints (mostly for 2 hours). Six (17.6\%) of 34 deaths were caused by PE, four of which were confirmed by autopsy. None of the four patients had a medical or premorbid condition associated with VT or causing prolonged immobilisation. Only two patients were taking long-term antipsychotics, but all were physically restrained and collapsed suddenly shortly before the PE event.

\section{Case Presentation}

\section{Case 1}

In October 2010, a 61-year-old man with schizophrenia was admitted for disturbing behaviour. He was a chronic smoker with no history of alcohol or substance abuse. He was given lithium, risperidone, quetiapine, clonazepam, and haloperidol decanoate. In addition, he was given nitrofurantoin for urinary tract infection and haloperidol (5 $\mathrm{mg}$ ) injection for sedation, and was physically restrained on day 1 and day 9. On day 11, he collapsed suddenly and died 1 hour later despite cardiopulmonary resuscitation. Autopsy showed PE and DVT of both calves.

\section{Case 2}

In February 2012, an 86-year-old man with Alzheimer dementia was admitted for aggressive behaviour. He was disturbing and shouting and was physically restrained five times for 17 hours in 2 days. On day 1 , he was treated for hypokalaemia $(2.8 \mathrm{mmol} / \mathrm{L})$, which was corrected to $4.1 \mathrm{mmol} / \mathrm{L}$ the next day. On day 2 , his serum creatinine level rose to $187 \mathrm{mmol} / \mathrm{L}$ suggesting dehydration. He was given venlafaxine, valproate, and risperidone. On day 4 , his condition rapidly deteriorated and he died 1 hour later. Autopsy revealed massive PE and DVT of the legs.

\section{Case 3}

In December 2013, a 43-year-old African man with bipolar disorder who did not take medication regularly was found wandering. He was mute and uncooperative and refused 
examination in the emergency room. He was treated in a medical ward for 2 days and then was restrained for an unspecified period. Toxicology screening test was negative. He was transferred to a psychiatric hospital. He collapsed suddenly and died 1 hour after arrival. Autopsy revealed coils of thromboemboli inside the pulmonary arteries that had occurred at least months before and DVT of both legs. The coroner concluded that he had recurrent DVT causing mild PE months earlier before this fatal PE.

\section{Case 4}

In February 2014, a 46-year-old woman with schizophrenia and beta-thalassaemia was admitted for poor sleep, decreased appetite, feeling of being watched, and refusal to talk. She had refused psychiatric follow-up and medication. Both blood and urine toxicology screening tests were negative. She was given two injections of haloperidol and was physically restrained for 10 hours in 2 days. On day 3 , she collapsed suddenly and died despite 1 hour of resuscitation. Autopsy showed numerous thromboemboli in branches of main pulmonary arteries completely occluding the lumen despite no DVT.

\section{Discussion}

In Hong Kong, physical restraint is widely practised in psychiatric and general hospitals, but its dire consequences are often under-recognised. Our case reports suggest that physical restraints seem to be associated with PE. Therefore, we suggest using manual restraint by staff, talk-down and de-escalation techniques, or rapid-tranquillisation drugs in managing acutely disturbing patients. If physical restraint is deemed unavoidable, the patient's hydration status should be monitored, particularly when patients have no fluid intake after a long period of shouting or crying, and more active or passive physical exercises should be allowed. Physical restraint should be used with caution, especially in patients on long-term antipsychotics or those with medical conditions that affect blood coagulation. As fatal PE can occur suddenly, we suggest using Doppler ultrasonography, magnetic resonance imaging, or D-dimer test to detect the condition earlier. Anticoagulation prophylaxis (such as subcutaneous heparin, elastic stockings, and low molecular heparin) can be given to high-risk patients. In 65 of 170 physically restraint or secluded patients who were administered anticoagulants, none developed DVT. ${ }^{7}$

\section{Declaration}

The authors have no conflict of interest to disclose.

\section{References}

1. Cheuk BL, Cheung GC, Cheng SW. Epidemiology of venous thromboembolism in a Chinese population. Br J Surg 2004;91:4248. Crossref

2. Chau KY, Yuen ST, Wong MP. Clinicopathological pattern of pulmonary thromboembolism in Chinese autopsy patients: comparison with Caucasian series. Pathology 1997;29:263-6. Crossref

3. Thomassen R, Vandenbroucke JP, Rosendaal FR. Antipsychotic medication and venous thrombosis. Br J Psychiatry 2001;179:636. Crossref

4. Allenet B, Schmidlin S, Genty C, Bosson JL. Antipsychotic drugs and risk of pulmonary embolism. Pharmacoepidemiol Drug Saf 2012;21:42-8. Crossref

5. Hem E, Steen O, Opjordsmoen S. Thrombosis associated with physical restraints. Acta Psychiatr Scand 2001;103:73-6. Crossref

6. WWK Wu. Psychosocial correlates of patients being physically restrained within the first 7 days in an acute psychiatric admission ward: retrospective case record review. East Asian Arch Psychiatry 2015;25:47-57.

7. De Herti M, Einfinger G, Scherpenberg E, Wampers M, Peuskens J. The prevention of deep venous thrombosis in physically restrained patients with schizophrenia. Int J Clin Pract 2010;64:1109-15. Crossref 